\title{
A turnpike theorem involving a modified Golden Rule
}

\author{
Darong DAI \\ Nanjing University, China \\ daidarong998@163.com \\ Kunrong SHEN \\ Nanjing University, China \\ shenkr@nju.edu.cn
}

\begin{abstract}
In the current study, we investigate efficient capital accumulation in a stochastic neoclassical aggregate growth model. The underlying uncertainty is driven by Brownian-motion shocks and the major results do not rely on the specification of production functions. The stochastic balanced path of the capitallabor ratio is naturally derived by a martingale, and the corresponding modified Golden Rule path of capital accumulation is well-defined. The powerful martingale theory is thus employed, and a stochastic turnpike theorem involving the modified Golden Rule is proved. That is, the underlying path of capital accumulation is asymptotically efficient in the sense of consumption maximization. We focus on asymptotic turnpike theorems and our turnpike theorem only relies on the requirement that the modified Golden-Rule path of capital accumulation is reachable in any almost surely finite Markov time. Finally, it is asserted that the modified Golden-Rule path of capital accumulation indeed provides us with a robust turnpike under very weak assumptions.
\end{abstract}

Keywords: capital accumulation; stochastic balanced path; martingale path; modified golden rule; turnpike theorem; robustness.

JEL Classification: C60, E13, E22.

REL Classification: 8E, 10Z. 


\section{Introduction}

In his seminal paper, Merton (1975) extends the one-sector neoclassical growth model of Solow-type to stochastic cases where the dynamics of capital-labor ratio is driven by a diffusion process, thereby providing us with an asymptotic theory of economic growth under uncertainty. Later on, Chang and Malliaris (1987) prove a theorem that confirms the existence and uniqueness of the stochastic growth path derived by Merton under certain assumptions. Therefore, noting the important and interesting properties reflected by Merton's model, the motivation of present exploration is to derive a well-defined modified Golden Rule path of capital accumulation and establish corresponding turnpike theorem based upon Merton's framework and also the theorem demonstrated by Chang and Malliaris. In other words, the current study enriches Merton's model and conclusion by uncovering a robust turnpike theorem involving the modified Golden Rule implicitly implied by the basic model.

In deterministic neoclassical models, Golden Rule or modified Golden Rule is usually derived through the balanced path of capital-labor ratio (Cass, 1966, 1972). Similarly, the present modified Golden Rule is established via letting the drift term of the diffusion process of capital-labor ratio be equal to zero, thereby producing a martingale path of capital accumulation. That is, we define the stochastic balanced path of capital-labor ratio of the current stochastic neoclassical model by the martingale-path of capital-labor ratio. As a matter of fact, there is a natural one-to-one correspondence between the modified Golden Rule and the martingale-path of capital-labor ratio. Consequently, it is ensured that the modified Golden Rule derived through the stochastic balanced path, i.e., the martingale path, of capital-labor ratio is well defined. And this creates a natural opportunity such that the powerful martingale theory can be appropriately employed to demonstrate the turnpike theorem. Rather, the present turnpike theorem shows that the martingale-path of capital-labor ratio will converge to the modified Golden Rule almost surely and in the sense of uniform topology (Dai, 2012) as long as the modified Golden Rule is reachable in any almost surely finite time. And one can easily notice the differences between the present result and those proved by Cass (1966) and Samuelson (1965) in deterministic neoclassical aggregate growth models.

When we define the concept of capital in a very broad sense, i.e., including human capital, health capital, environmental capital, and so on, then capital accumulation indeed plays a crucial role in modern economic growth. For example, the Germany and Japan after World War II and China after 1978s (Song et al., 2011). We, hence, are motivated to explore efficient capital accumulation in stochastic growth economies with Brownian-motion shocks. The major contribution of the 
present paper can be summarized as follows: first, since Brownian motion shocks are widely used in continuous-time stochastic growth models, we provide an appropriate definition of the modified Golden-Rule path of capital accumulation in such kind of economies and this definition does not rely on the explicit specification of the Brownian shocks; second, we develop a systematic mathematical method for proving robust turnpike theorems in such kind of circumstances involving the above modified Golden-Rule path of capital accumulation, and we believe that our method is general enough to be used in other related environments. In other words, our contribution mainly focuses on theoretical issues of macroeconomic growth theory.

There are some related literatures. As is argued by Yano (1985), existing turnpike theorems in optimal growth theory can be summarized as the following two types, one is neighborhood turnpike theorem (Yano, 1984, McKenzie, 1982, Kamihigashi, Roy, 2007, Kondo, 2008, Dai, 2012) which asserts that an optimal path in a growth model converges to a small neighborhood of a stationary path, the other is asymptotic turnpike theorem (Bewley, 1982, Yano, 1985, Sahashi, 2002, Dai, 2012) which means that an optimal path converges to a stationary path. As you will see below, the present paper focuses on asymptotic turnpike theorem and we have confirmed the corresponding robustness in a continuous-time stochastic growth model. On the other hand, it is well-known that the Golden Rule path has been playing a very important role in neoclassical theory of capital accumulation (Cass, 1966, 1972, Samuelson, 1965, de la Croix and Ponthiere, 2010, Mitra, Ray, 2012, Acemoglu, 2012) starting from the pioneering papers of Phelps (1962, 1965). Recently, Schenk-Hoppé (2002) also studies the Golden Rule in stochastic Solow growth model. Schenk-Hoppé employs dynamical systems theory, especially the concept of a random fixed point (Schenk-Hoppé, Schmalfuss, 2001), to prove the existence of a Golden-Rule savings rate for the stochastic Solow model. Methodologically, in studying the Golden-Rule path of capital accumulation, Brock and Mirman (1972) use the classical stochastic stability theory of Markov chains while Bayer and Wälde (2011) expand their distributional analysis by using the stability theory for Markov processes in continuous time. We heavily employ martingale theory, which depends on continuous-time Markov processes driven by Brownian motions in the present economy, to demonstrate the corresponding turnpike theorem involving the modified Golden-Rule path of capital accumulation. This method can be regarded as a useful complement to existing literatures involving the issue of efficient capital accumulation under uncertainty.

The rest of the paper is organized as follows. Section 2 presents the basic model. Section 3 defines the modified Golden Rule path of capital accumulation, proves the turnpike theorem and also confirms the corresponding robustness. Section 4 
closes the paper with some concluding remarks. All proofs, unless otherwise noted in the text, appear in the Appendix.

\section{The model}

The major goal of the model is to introduce the stochastic path of capital accumulation in a one-sector neoclassical growth model with the uncertainty coming from the population size $L(t)$, i.e., following Merton (1975),

$d L(t)=n L(t) d t+\sigma L(t) d B(t)$.

which is based upon the underlying filtered probability space $\left(\Omega, \mathrm{F},\left\{\mathrm{F}_{t}\right\}_{0 \leq t \leq T}, \mathrm{P}\right)$ with $\mathrm{E}$ denoting the expectation operator depending on $\mathrm{F}_{0} \triangleq\{\Omega, \varnothing\}$.

As usual, the neoclassical production function $Y(t)=F(K(t), L(t))$ is assumed to be strictly concave, homogeneous of first degree and also exhibit constant returns to scale with the law of motion of capital accumulation expressed as follows,

$\frac{d K(t)}{d t}=F(K(t), L(t))-\delta K(t)-C(t)$.

in which, $\delta$, an exogenously given constant, denotes the depreciation rate and $C(t)$ represents aggregate consumption at time $t$.

Now, combining (1) with (2) and applying the classical Itô's rule yields the following SDE of capital-labor ratio,

$d k(t)=\left[f(k(t))-\left(\delta+n-\sigma^{2}\right) k(t)-c(t)\right] d t-\sigma k(t) d B(t)$.

subject to $k(0) \equiv k_{0}>0$, a deterministic constant. And $f(k(t)) \triangleq Y(t) / L(t), c(t)$

$\triangleq C(t) / L(t)$ stand for per capita output and per capita consumption, respectively, at time $t$. Specifically, for the SDE of capital-labor ratio given by (3), Chang and Malliaris (1987) proved the following result,

PROPOSITION 1: If the production function $f$ is strictly concave, continuously differentiable on $[0, \infty), f(0)=0$, and $\lim _{k(t) \rightarrow \infty} f^{\prime}(k(t)) \triangleq \lim _{k(t) \rightarrow \infty} \frac{d f(k(t))}{d k(t)}=0$, then there exists a unique solution to (3).

In order to make things much easier, we need,

ASSUMPTION 1: The assumptions or conditions given by Proposition 1 are assumed to be fulfilled throughout the current paper. 


\section{Turnpike theorem}

In the present section, we will derive a modified Golden Rule and establish the corresponding turnpike theorem under relatively weak conditions. For the SDE of capital-labor ratio given by (3), we denote the drift term by,

$b(t) \triangleq f(k(t))-\left(\delta+n-\sigma^{2}\right) k(t)-c(t)$.

which implies that the capital-labor ratio $k(t)$ tends to increase if $b(t)>0$, and the capital-labor ratio tends to decrease if $b(t)<0$. Noting that the Golden Rule or modified Golden Rule is usually derived via the balanced path of capital-labor ratio in the deterministic case (Cass, 1966, 1972), we similarly derive the modified Golden Rule by letting $b(t)=0$, which corresponds to the stochastic balanced path of capital-labor ratio and this gives rise to,

$c(t)=f(k(t))-\left(\delta+n-\sigma^{2}\right) k(t)$.

Hence, the corresponding stochastic Golden Rule $k^{*}$ is determined by,

$f^{\prime}\left(k^{*}\right)=\delta+n-\sigma^{2}$.

Meanwhile, substituting (5) into (3) leads us to,

$d k(t)=-\sigma k(t) d B(t)$.

which hence defines a martingale-path of capital-labor ratio. Now, we can establish,

THEOREM 1 (Turnpike Theorem): If the following Markov time,

$$
\tau^{*}(\omega) \triangleq \inf \left\{t \geq 0 ; k(t)=k^{*}\right\}<\infty \text { a.s. }
$$

then we get that the martingale-path of capital-labor ratio given by (7) will strongly converge to the stochastic Golden Rule $k^{*}$ given by (6) a.s. and in the sense of uniform topology.

PROOF: By the Doob's Martingale Inequality,

$\mathrm{P}\left(\sup _{0 \leq t \leq T}|k(t)| \geq \lambda\right) \leq \frac{1}{\lambda} \mathrm{E}[|k(T)|]=\frac{k_{0}}{\lambda}, \forall \lambda>0, \forall T>0$.

Without loss of generality, we put $\lambda=2^{m}$ for $m \in \mathrm{N}$, then,

$\mathrm{P}\left(\sup _{0 \leq t \leq T}|k(t)| \geq 2^{m}\right) \leq \frac{1}{2^{m}} k_{0}, \forall m \in \mathrm{N}, \forall T>0$.

Using the well-known Borel-Cantelli Lemma, we arrive at, 
$\mathrm{P}\left(\sup _{0 \leq t \leq T}|k(t)| \geq 2^{m}\right.$ for infinitely many $\left.m\right)=0, \forall T>0$.

So for a.a. $\omega \in \Omega$, there exists $\bar{m}(\omega) \in \mathrm{N}$ such that,

$\sup _{0 \leq t \leq T}|k(t)|<2^{m}$ a.s. for $m \geq \bar{m}(\omega), \forall T>0$.

i.e.,

$\lim _{T \rightarrow \infty} \sup _{0 \leq t \leq T}|k(t)| \leq 2^{m}$ a.s. for $m \geq \bar{m}(\omega)$.

Thus, $k(t)=k(t, \omega)$ is uniformly bounded for $t \in[0, T], \forall T>0$ and for a.a. $\omega \in \Omega$. Define,

$B_{2^{-m}}\left(\tau^{*}(\omega)\right) \triangleq\left\{\tau(\omega) \geq 0 ;\left|\tau(\omega)-\tau^{*}(\omega)\right|<2^{-m}\right\} \forall m \in \mathrm{N}$.

Thus, for $\forall \tau^{m} \in B_{2^{-m}}\left(\tau^{*}\right)$, and based on the assumption that $\tau^{*}(\omega)<\infty$ a.s., applying Doob's optional sampling theorem and Doob's martingale inequality lead us to,

$\mathrm{P}\left(\sup _{0 \leq t \leq \tau^{m}}\left|k(t)-k^{*}\right| \geq \varepsilon\right) \leq \frac{1}{\varepsilon} \mathrm{E}\left[\left|k\left(\tau^{m}\right)-k^{*}\right|\right], \forall \varepsilon>0$.

According to (8) and the continuity of martingale w. r. t. time $t$, an application of Lebesgue bounded convergence theorem shows,

$\limsup _{m \rightarrow \infty} \mathrm{P}\left(\sup _{0 \leq t \leq \tau^{m}}\left|k(t)-k^{*}\right| \geq \varepsilon\right) \leq \frac{1}{\varepsilon} \limsup _{m \rightarrow \infty} \mathrm{E}\left[\left|k\left(\tau^{m}\right)-k^{*}\right|\right]=0, \forall \varepsilon>0$.

which yields,

$\limsup _{m \rightarrow \infty} \mathrm{P}\left(\sup _{0 \leq t \leq \tau^{m}}\left|k(t)-k^{*}\right|<\varepsilon\right)=1, \forall \varepsilon>0$.

It follows from Fatou's Lemma that,

$\mathrm{P}\left(\sup _{0 \leq t \leq \tau^{*}}\left|k(t)-k^{*}\right|<\varepsilon\right)=1, \forall \varepsilon>0$.

Thus, we get,

$\sup _{0 \leq t \leq \tau^{*}}\left|k(t)-k^{*}\right|<\varepsilon$, a.s. for $\forall \varepsilon>0$.

i.e.,

$\underset{\tau^{*} \rightarrow \infty}{\lim \sup } \sup _{0 \leq t \leq \tau^{*}}\left|k(t)-k^{*}\right|<\varepsilon$, a.s. for $\forall \varepsilon>0$.

Noting the arbitrariness of $\varepsilon$, the required assertion follows. 
Next, we proceed to analyze the robustness of the turnpike theorem given by Theorem 1, i.e., we show that the modified Golden Rule $k^{*}$ indeed provides us with a robust turnpike under relatively weak assumptions. Based upon the martingale path given by (7), we set,

$d \tilde{k}(t)=-\tilde{\sigma} \tilde{k}(t) d B(t)$.

subject to $\tilde{k}(0) \triangleq k_{0}>0$, a deterministic constant, such that,

ASSUMPTION 2: For any $\xi>0$, suppose that,

$|\sigma-\tilde{\sigma}| \leq \xi$.

for any non-zero constants $\sigma$ and $\tilde{\sigma}$ with $|\sigma| \vee|\tilde{\sigma}|<\infty$.

As preparations, we need the following two lemmas,

LEMMA 1: There exist constants $e\left(k_{0}, p, T\right)<\infty$ and $\tilde{e}\left(k_{0}, p, T\right)<\infty$ such that,

(i) $\mathrm{E}\left[\sup _{0 \leq t \leq T}|k(t)|^{p}\right] \leq e\left(k_{0}, p, T\right)$.

and,

(ii) $\mathrm{E}\left[\sup _{0 \leq t \leq T}|\tilde{k}(t)|^{p}\right] \leq \tilde{e}\left(k_{0}, p, T\right)$.

for $\forall 0<T<\infty, \forall p \in \mathrm{N}, p \geq 2$.

PROOF: See Appendix A.

Rather, we can obtain the following result for the present case,

LEMMA 2: For $k(t)$ defined in (7) and $\tilde{k}(t)$ defined in (9), one can get that,

(i) $\mathrm{E}\left[\sup _{0 \leq t \leq T}|k(t)|^{p}\right] \leq\left(\frac{p}{p-1}\right)^{p} \lambda^{p-1} k_{0}$.

and,

(ii) $\mathrm{E}\left[\sup _{0 \leq t \leq T}|\tilde{k}(t)|^{p}\right] \leq\left(\frac{p}{p-1}\right)^{p} \tilde{\lambda}^{p-1} k_{0}$.

for $\forall 0<\lambda<\infty, \forall 0<\tilde{\lambda}<\infty, \forall 0<T<\infty$, and $\forall p \in \mathrm{N}, p \geq 2$.

PROOF: See Appendix B. 
Then, the following proposition is derived,

PROPOSITION 2: Based upon the above assumptions and Lemma 1 or 2, then we have,

$\mathrm{E}\left[\lim _{T \rightarrow \infty} \sup _{0 \leq t \leq T}|k(t)-\tilde{k}(t)|^{2}\right] \rightarrow 0$ as $\xi \rightarrow 0$.

PROOF: See Appendix C.

As you can see in the proof of Proposition 2, Lemma 1 or Lemma 2 plays a technical role in confirming the corresponding stability of the underlying turnpike. Indeed, Lemma 1 proves the uniform bound property of the path of capital accumulation and we have further provided the explicit supremum of the path of capital accumulation in Lemma 2, which imply that both Lemma 1 and Lemma 2 are of independent interest in characterizing intrinsic properties of the underlying path of capital accumulation. Consequently, combining Theorem 1 with Proposition 2 reveals that,

COROLLARY 1 (Robust Turnpike Theorem): Provided Theorem 1 and Proposition 2, we get that the turnpike theorem keeps invariant as the perturbation $\xi \rightarrow 0$, i.e., the modified Golden Rule $k^{*}$ is indeed a robust turnpike in the present model economy.

\section{Concluding remarks}

As is broadly known, both turnpike theorems and the Phelps-Koopmans Theorem play very important roles in macroeconomics (Acemoglu, 2012). Turnpike theory (McKenzie, 1976, 1998, Joshi, 1997, Dai, 2012) characterizes the mathematical properties of the equilibrium or optimal path of resource allocation while the classical Phelps-Koopmans Theorem (Phelps, 1962, 1965, Ray, 2010, Mitra, Ray, 2012) clearly uncovers that the efficient path of capital accumulation will definitely converge to the Golden Rule in the long run, otherwise, dynamically inefficient accumulation happens. Samuelson (1965) proved a neighborhood turnpike theorem involving the Golden Rule in the classical Ramsey (1928) model, while the present paper demonstrates an asymptotic turnpike theorem involving the modified Golden Rule in a stochastic neoclassical growth model, which implies that the path of capital accumulation is dynamically efficient. Finally, it is also confirmed that the modified Golden Rule path of capital accumulation is indeed a robust turnpike.

Finally, noting that the present paper just represents a simple starting point in exploring turnpike properties of capital accumulation from the present perspective, some interesting extensions can be taken into account in future 
research. For example, since we only proves an asymptotic turnpike theorem in the underlying economy, one interesting and possible extension is to find out conditions supporting a neighborhood turnpike theorem for neighborhood efficiency characterization of stochastic capital accumulation. Moreover, notice that the (modified) Ramsey rule also plays a crucial role in savings behavior and macroeconomic growth, it is interesting to investigate the corresponding turnpike theorems involving Ramsey rules by effectively employing the mathematical method developed in the present study. As a final point, if we are motivated to investigate the effect of stochastic TFP imposed on the efficient path of capital accumulation, geometric Brownian motion can be naturally employed with the purpose of introducing technology fluctuation into the underlying economy as in Wälde (2011) and Bucci et al. (2011).

\section{References}

Acemoglu, D. (2012). "Introduction to Economic Growth", Journal of Economic Theory, 147, pp. $545-550$

Bayer, C., Wälde, K. (2011). "Existence, Uniqueness and Stability of Invariant Distributions in Continuous-Time Stochastic Models", Working paper

Bewley, T. (1982). "An Integration of Equilibrium Theory and Turnpike Theory", Journal of Mathematical Economics, 10, pp. 233-267

Brock, W.A., Mirman L.J. (1972). "Optimal Economic Growth and Uncertainty: The Discounted Case”, Journal of Economic Theory, 4, pp. 479-513

Bucci, A., Colapinto, C., Forster, M., Torre, D.L. (2011). "Stochastic Technology Shocks in an Extended Uzawa-Lucas Model: Closed-Form Solution and Long-Run Dynamics”, Journal of Economics, 103, pp. 83-99

Cass, D. (1966). "Optimum Growth in an Aggregative Model of Capital Accumulation: A Turnpike Theorem", Econometrica, 34, pp. 833-850

Cass, D. (1972). "On Capital Overaccumulation in the Aggregative, Neoclassical Model of Economic Growth: A Complete Characterization", Journal of Economic Theory, 4, pp. 200-223

Chang, F.-R., Malliaris, A.G. (1987). "Asymptotic Growth under Uncertainty: Existence and Uniqueness", Review of Economic Studies, 54, pp. 169-174

Dai, D. (2012). "Stochastic Versions of Turnpike Theorems in the Sense of Uniform Topology", Annals of Economics and Finance, 13, pp. 389-431

de la Croix, D., Ponthiere, G. (2010). "On the Golden Rule of Capital Accumulation under Endogenous Longevity”, Mathematical Social Sciences, 59, pp. 227-238

Higham, D.J., Mao, X.R., Stuart, A.M. (2003). "Strong Convergence of Euler-Type Methods for Nonlinear Stochastic Differential Equations", SIAM Journal on Numerical Analysis, 40, pp. 1041-1063

Joshi, S. (1997). "Turnpike Theorem in Nonconvex and Nonstationary Environment", International Economic Review, 38, pp. 225-248 
Kamihigashi, T., Roy, S. (2007). “A Nonsmooth, Nonconvex Model of Optimal Growth”, Journal of Economic Theory, 132, pp. 435-460

Karatzas, I. Shreve, S.E. (1991). "Brownian Motion and Stochastic Calculus", Graduate Texts in Mathematics, New York: Springer-Verlag, second edition

Kondo, A. (2008). "On the Inefficacy of Temporary Policy in a Dynamic General Equilibrium with Money", Japanese Economic Review, 59, pp. 324-344

McKenzie, L. (1976). “Turnpike Theory”, Econometrica, 44, pp. 841-865

McKenzie, L. (1982). "A Primal Route to the Turnpike and Liapounov Stability", Journal of Economic Theory, 27, pp. 194-209

McKenzie, L. (1998). "Turnpikes”, American Economic Review, 88, pp. 1-14

Merton, R.C. (1975). "An Asymptotic Theory of Growth under Uncertainty", Review of Economic Studies, 42, pp. 375-393

Mitra, T., Ray, D. (2012). "On the Phelps-Koopmans Theorem", Journal of Economic Theory, 147 , pp. $833-849$

Phelps, E.S. (1962). "The End of the Golden Age in Solovia: Comment", American Economic Review, 52, pp. 1097-1099

Phelps, E.S. (1965). "Second Essay on the Golden Rule of Accumulation", American Economic Review, 55, pp. 793-814

Ramsey, F.P. (1928). "A Mathematical Theory of Saving”, Economic Journal, 38, pp. 543-559

Ray, D. (2010). "The Phelps-Koopmans Theorem and Potential Optimality", International Journal of Economic Theory, 6, pp. 11-28

Sahashi, Y. (2002). "The Convergence of Optimal Forestry Control", Journal of Mathematical Economics, 37, pp. 179-214

Samuelson, P.A. (1965). "A Catenary Turnpike Theorem Involving Consumption and the Golden Rule", American Economic Review, 55, pp. 486-496

Schenk-Hoppé, K.R. (2002). "Is There A Golden Rule For The Stochastic Solow Growth Model?", Macroeconomic Dynamics, 6, pp. 457-475

Schenk-Hoppé, K.R., Schmalfuss, B. (2001). "Random Fixed Points in a Stochastic Solow Growth Model", Journal of Mathematical Economics, 36, pp. 19-30

Song, Z., Storesletten, K., Zilibotti, F. (2011). "Growing Like China", American Economic Review, 101, pp. 202-241

Wälde, K. (2011). "Production Technologies in Stochastic Continuous Time Models", Journal of Economic Dynamics and Control, 35, pp. 616-622

Yano, M. (1984). "Competitive Equilibria on Turnpikes in a McKenzie Economy, I: A Neighborhood Turnpike Theorem", International Economic Review, 25, pp. 695-717

Yano, M. (1985). "Competitive Equilibria on Turnpikes in a McKenzie Economy, II: An Asymptotic Turnpike Theorem", International Economic Review, 26, pp. 661-669 


\section{Appendix}

\section{A. Proof of Lemma 1}

Applying Itô's rule to (7),

$|k(t)|^{2}=k_{0}^{2}+\int_{0}^{t} \sigma^{2} k^{2}(s) d s+2 \int_{0}^{t}\left(-\sigma k^{2}(s)\right) d B(s)$.

Thus, for $\forall t_{1} \in[0, T]$ and for some constant $e=e(p)$, which may be different from line to line throughout the proof, we get,

$$
\sup _{0 \leq t \leq t_{1}}|k(t)|^{p} \leq e\left\{k_{0}^{p}+\left[\int_{0}^{t_{1}} \sigma^{2} k^{2}(s) d s\right]^{\frac{p}{2}}+\sup _{0 \leq t \leq t_{1}}\left|\int_{0}^{t}\left(-\sigma k^{2}(s)\right) d B(s)\right|^{\frac{p}{2}}\right\} .
$$

It follows from Cauchy-Schwarz Inequality that,

$$
\sup _{0 \leq t \leq t_{1}}|k(t)|^{p} \leq e\left\{k_{0}^{p}+\int_{0}^{t_{1}}|k(s)|^{p} d s+\sup _{0 \leq t \leq t_{1}}\left|\int_{0}^{t}\left(-\sigma k^{2}(s)\right) d B(s)\right|^{\frac{p}{2}}\right\} \text {. }
$$

Taking expectations on both sides and applying the Burkholder-Davis-Gundy Inequality (see, Karatzas Shreve, 1991, p. 166) shows,

$$
\mathrm{E}\left[\sup _{0 \leq t \leq t_{1}}|k(t)|^{p}\right] \leq e\left\{k_{0}^{p}+\int_{0}^{t_{1}} \mathrm{E}|k(s)|^{p} d s+\mathrm{E}\left[\int_{0}^{t_{1}} k^{4}(s) d s\right]^{\frac{p}{4}}\right\} .
$$

By the Young Inequality (Higham et al., 2003) and Rogers-Hölder Inequality,

$$
\begin{aligned}
& \mathrm{E}\left[\int_{0}^{t_{1}} k^{4}(s) d s\right]^{\frac{p}{4}} \\
& \leq \mathrm{E}\left[\sup _{0 \leq t \leq t_{1}}|k(t)|^{\frac{p}{2}}\left(\int_{0}^{t_{1}} k^{2}(s) d s\right)^{\frac{p}{4}}\right] \\
& \leq \frac{1}{2 e} \mathrm{E}\left[\sup _{0 \leq t \leq t_{1}}|k(t)|^{p}\right]+\frac{e}{2} \mathrm{E}\left[\int_{0}^{t_{1}} k^{2}(s) d s\right]^{\frac{p}{2}} \\
& \leq \frac{1}{2 e} \mathrm{E}\left[\sup _{0 \leq t \leq t_{1}}|k(t)|^{p}\right]+e \mathrm{E}\left[\int_{0}^{t_{1}}|k(s)|^{p} d s\right] .
\end{aligned}
$$

Substituting this into (A.1) yields, 
$\mathrm{E}\left[\sup _{0 \leq t \leq t_{1}}|k(t)|^{p}\right] \leq e\left\{k_{0}^{p}+\int_{0}^{t_{1}} \mathrm{E}|k(s)|^{p} d s\right\}$.

Applying Itô's rule to (7) produces,

$k(t)=k_{0} \exp \left(-\sigma B(t)-\frac{1}{2} \sigma^{2} t\right)$.

which implies,

$\mathrm{E}\left[|k(t)|^{p}\right]=k_{0}^{p} \exp \left(\frac{1}{2} \sigma^{2} p(p-1) t\right)$.

by the Wald's Identity. By (A.3), we hence get,

$\int_{0}^{t_{1}} \mathrm{E}|k(s)|^{p} d s=\frac{2 k_{0}^{p}}{\sigma^{2} p(p-1)}\left[\exp \left(\frac{1}{2} \sigma^{2} p(p-1) t_{1}\right)-1\right]$.

Inserting this into (A.2) reveals that there exists a constant $e\left(k_{0}, p, T\right)<\infty$ such that for $\forall 0<T<\infty$, $\mathrm{E}\left[\sup _{0 \leq t \leq T}|k(t)|^{p}\right] \leq e\left(k_{0}, p, T\right)$.

as required in (i). Noting that the proof of (ii) is quite similar to that of (i), we omit it. And thus the proof is complete.

\section{B. Proof of Lemma 2}

By Doob's martingale inequality,

$$
\mathrm{P}\left(\sup _{0 \leq t \leq T}|k(t)| \geq \lambda\right) \leq \frac{1}{\lambda} \mathrm{E}[|k(T)|]=\frac{k_{0}}{\lambda}, \forall 0<\lambda<\infty, \forall T>0 .
$$

Using Doob's martingale inequality again shows,

$$
\mathrm{P}\left(\sup _{0 \leq t \leq T}|k(t)| \geq \lambda\right) \leq \frac{1}{\lambda^{p}} \mathrm{E}\left[|k(T)|^{p}\right], \forall 0<\lambda<\infty, \forall T>0, \forall p \in \mathrm{N}, p \geq 2 .
$$

which combines with (B.1) produces,

$$
\frac{1}{\lambda^{p}} \mathrm{E}\left[|k(T)|^{p}\right] \leq \frac{k_{0}}{\lambda} \Leftrightarrow \mathrm{E}\left[|k(T)|^{p}\right] \leq \lambda^{p-1} k_{0}, \forall 0<\lambda<\infty, \forall T>0 .
$$

Define,

$$
\Sigma^{*} \triangleq \sup _{0 \leq t \leq T}|k(t)|,\|\Sigma\|_{p} \triangleq\|k(T)\|_{p} \triangleq\left\{\mathrm{E}\left[|k(T)|^{p}\right]\right\}^{\frac{1}{p}} .
$$

for $\forall T>0, \forall p \in \mathrm{N}, p \geq 2$. Let $H>0$ be some constant, then by Doob's Maximal Inequality and Fubini Theorem, we obtain, 


$$
\begin{aligned}
& \mathrm{E}\left[\left|\Sigma^{*} \wedge H\right|^{p}\right]=p \int_{0}^{\infty} x^{p-1} \mathrm{P}\left(\Sigma^{*} \wedge H \geq x\right) d x \leq p \int_{0}^{\infty} x^{p-2}\left(\int_{\left\{\Sigma^{*} \wedge H \geq x\right\}} \Sigma d \mathrm{P}\right) d x \\
& =p \int_{0}^{\infty} x^{p-2}\left(\int_{\Omega} \Sigma 1_{\left\{\Sigma^{*} \wedge H \geq x\right\}} d \mathrm{P}\right) d x=p \int_{\Omega} \Sigma\left(\int_{0}^{\Sigma^{*}} \hat{\Omega}^{H} x^{p-2} d x\right) d \mathrm{P} \\
& =\frac{p}{p-1} \mathrm{E}\left[\Sigma\left|\Sigma^{*} \wedge H\right|^{p-1}\right] .
\end{aligned}
$$

It follows from Hölder Inequality that,

$$
\left\|\Sigma^{*} \wedge H\right\|_{p}^{p}=\mathrm{E}\left[\left|\Sigma^{*} \wedge H\right|^{p}\right] \leq \frac{p}{p-1}\|\Sigma\|_{p}\left\|\left|\Sigma^{*} \wedge H\right|^{p-1}\right\|_{\frac{p}{p-1}}=\frac{p}{p-1}\|\Sigma\|_{p}\left\{\mathrm{E}\left[\left|\Sigma^{*} \wedge H\right|^{p}\right]\right\}^{\frac{p-1}{p}} .
$$

i.e.,

$$
\left\{\mathrm{E}\left[\left|\Sigma^{*} \wedge H\right|^{p}\right]\right\}^{\frac{1}{p}} \leq \frac{p}{p-1}\|\Sigma\|_{p}=\frac{p}{p-1}\left\{\mathrm{E}\left[|k(T)|^{p}\right]\right\}^{\frac{1}{p}} .
$$

Hence, applying Lebesgue Dominated Convergence Theorem shows,

$$
\mathrm{E}\left[\left|\Sigma^{*}\right|^{p}\right] \leq\left(\frac{p}{p-1}\right)^{p} \mathrm{E}\left[|k(T)|^{p}\right] \text {. }
$$

i.e.,

$$
\mathrm{E}\left[\sup _{0 \leq t \leq T}|k(t)|^{p}\right] \leq\left(\frac{p}{p-1}\right)^{p} \lambda^{p-1} k_{0} \forall 0<\lambda<\infty, \forall T>0, \forall p \in \mathrm{N}, p \geq 2 .
$$

by (B.2). And this gives the required result in (i). Noting that the proof of (ii) is similar to that of (i), we take it omitted here. And the proof is thus complete.

\section{Proof of Proposition 2}

By using Lemma 1 or 2 , there is a constant $W<\infty$ such that for $\forall T>0, \forall p \in \mathrm{N}$, $p \geq 2$,

$\mathrm{E}\left[\sup _{0 \leq t \leq T}|k(t)|^{p}\right] \vee \mathrm{E}\left[\sup _{0 \leq t \leq T}|\tilde{k}(t)|^{p}\right] \leq W$.

where by Assumption 1,

$$
k(t)=k_{0}+\int_{0}^{t}(-\sigma k(s)) d B(s) \text {. }
$$


$\tilde{k}(t)=k_{0}+\int_{0}^{t}(-\tilde{\sigma} \tilde{k}(s)) d B(s)$

Suppose that $|k(t)| \vee|\tilde{k}(t)| \leq \bar{W}$ for $t \geq 0$, otherwise, we just consider $k(t) \wedge \bar{W}$ and $\tilde{k}(t) \wedge \bar{W}$ instead of $k(t)$ and $\tilde{k}(t)$, respectively, for some constant $0<\bar{W}<\infty$. And also the same assertion follows by applying Lebesgue Dominated Convergence Theorem as $\bar{W}$ approaching infinity. In what follows, we first define the following stopping times,

$$
\tau_{\bar{W}} \triangleq \inf \{t \geq 0 ;|k(t)| \geq \bar{W}\}, \tilde{\tau}_{\bar{W}} \triangleq \inf \{t \geq 0 ;|\tilde{k}(t)| \geq \bar{W}\}, \hat{\tau}_{\bar{W}} \triangleq \tau_{\bar{W}} \wedge \tilde{\tau}_{\bar{W}} .
$$

By the Young Inequality (Higham et al., 2003) and for any $R>0$,

$$
\begin{aligned}
& \mathrm{E}\left[\sup _{0 \leq t \leq T}|k(t)-\tilde{k}(t)|^{2}\right] \\
& =\mathrm{E}\left[\sup _{0 \leq t \leq T}|k(t)-\tilde{k}(t)|^{2} 1_{\left\{\tau_{\bar{W}}>T, \tilde{\tau}_{\bar{W}}>T\right\}}\right]+\mathrm{E}\left[\sup _{0 \leq t \leq T}|k(t)-\tilde{k}(t)|^{2} 1_{\left\{\tau_{\bar{W}} \leq T, \text { or } \tilde{\tau}_{\bar{W}} \leq T\right\}}\right] \\
& \leq \mathrm{E}\left[\sup _{0 \leq t \leq T}\left|k\left(t \wedge \hat{\tau}_{\bar{W}}\right)-\tilde{k}\left(t \wedge \hat{\tau}_{\bar{W}}\right)\right|^{2} 1_{\left\{\hat{\tau}_{\bar{W}}>T\right\}}\right]+\frac{2 R}{p} \mathrm{E}\left[\sup _{0 \leq t \leq T}|k(t)-\tilde{k}(t)|^{p}\right] \\
& +\frac{1-\frac{2}{p}}{R^{\frac{2}{p-2}}} \mathrm{P}\left(\tau_{\bar{W}} \leq T, \text { or } \tilde{\tau}_{\bar{W}} \leq T\right) .
\end{aligned}
$$

It follows from (C.1) that,

$$
\mathrm{P}\left(\tau_{\bar{W}} \leq T\right)=\mathrm{E}\left[1_{\left\{\tau_{\bar{W}} \leq T\right\}} \frac{\left|k\left(\tau_{\bar{W}}\right)\right|^{p}}{\bar{W}^{p}}\right] \leq \frac{1}{\bar{W}^{p}} \mathrm{E}\left[\sup _{0 \leq t \leq T}|k(t)|^{p}\right] \leq \frac{W}{\bar{W}^{p}} .
$$

Similarly, one can get $\mathrm{P}\left(\tilde{\tau}_{\bar{W}} \leq T\right) \leq W / \bar{W}^{p}$. So,

$$
\mathrm{P}\left(\tau_{\bar{W}} \leq T, \text { or } \tilde{\tau}_{\bar{W}} \leq T\right) \leq \mathrm{P}\left(\tau_{\bar{W}} \leq T\right)+\mathrm{P}\left(\tilde{\tau}_{\bar{W}} \leq T\right) \leq \frac{2 W}{\bar{W}^{p}} .
$$

Noting that by (C.1),

$$
\mathrm{E}\left[\sup _{0 \leq t \leq T}|k(t)-\tilde{k}(t)|^{p}\right] \leq 2^{p-1} \mathrm{E}\left[\sup _{0 \leq t \leq T}\left(|k(t)|^{p}+|\tilde{k}(t)|^{p}\right)\right] \leq 2^{p} W \text {. }
$$

Hence, (C.2) can be rewritten as follows,

$$
\mathrm{E}\left[\sup _{0 \leq t \leq T}|k(t)-\tilde{k}(t)|^{2}\right]
$$


$\leq \mathrm{E}\left[\sup _{0 \leq t \leq T}\left|k\left(t \wedge \hat{\tau}_{\bar{W}}\right)-\tilde{k}\left(t \wedge \hat{\tau}_{\bar{W}}\right)\right|^{2}\right]+\frac{2^{p+1} R W}{p}+\frac{2(p-2) W}{p R^{\frac{2}{p-2} \bar{W}^{p}}}$.

Noting that,

$$
\begin{aligned}
& \left|k\left(t \wedge \hat{\tau}_{\bar{W}}\right)-\tilde{k}\left(t \wedge \hat{\tau}_{\bar{W}}\right)\right|^{2}=\left|\int_{0}^{t \wedge \hat{\tau}_{\bar{W}}}[(-\sigma) k(s)-(-\tilde{\sigma}) \tilde{k}(s)] d B(s)\right|^{2} \\
& =\left|\int_{0}^{t \wedge \hat{\tau}_{\bar{W}}}[(-\sigma) k(s)-(-\sigma) \tilde{k}(s)] d B(s)+\int_{0}^{t \wedge \hat{\bar{W}}_{\bar{W}}}[(-\sigma) \tilde{k}(s)-(-\tilde{\sigma}) \tilde{k}(s)] d B(s)\right|^{2} \\
& \leq 2\left(\sigma^{2}\left|\int_{0}^{t \wedge \hat{\bar{W}}_{\overline{\bar{W}}}}[k(s)-\tilde{k}(s)] d B(s)\right|^{2}+\left|\int_{0}^{t \wedge \hat{\bar{W}}_{\overline{\bar{W}}}}[(\tilde{\sigma}-\sigma) \tilde{k}(s)] d B(s)\right|^{2}\right) .
\end{aligned}
$$

Taking expectations on both sides and using Itô's Isometry, we have for $\forall \tau \leq T$,

$$
\begin{aligned}
& \mathrm{E}\left[\sup _{0 \leq t \leq \tau}\left|k\left(t \wedge \hat{\tau}_{\bar{W}}\right)-\tilde{k}\left(t \wedge \hat{\tau}_{\bar{W}}\right)\right|^{2}\right] \\
& \leq 2 \sigma^{2} \mathrm{E}\left[\int_{0}^{t \wedge \hat{\tau}_{\bar{W}}}(k(s)-\tilde{k}(s))^{2} d s\right]+2(\tilde{\sigma}-\sigma)^{2} \mathrm{E}\left[\int_{0}^{\tau}(\tilde{k}(s))^{2} d s\right] \\
& \leq 2 \sigma^{2} \int_{0}^{\tau} \mathrm{E}\left[\sup _{0 \leq t_{0} \leq s}\left|k\left(t_{0} \wedge \hat{\tau}_{\bar{W}}\right)-\tilde{k}\left(t_{0} \wedge \hat{\tau}_{\bar{W}}\right)\right|^{2}\right] d s+2 \xi^{2} \int_{0}^{\tau} \mathrm{E}|\tilde{k}(s)|^{2} d s .
\end{aligned}
$$

where we have used Assumption 2. Applying Itô's rule to (9) produces, $\tilde{k}(t)=k_{0} \exp \left(-\tilde{\sigma} B(t)-\frac{1}{2} \tilde{\sigma}^{2} t\right)$.

which implies,

$$
\mathrm{E}\left[|\tilde{k}(t)|^{2}\right]=k_{0}^{2} \exp \left(\tilde{\sigma}^{2} t\right)
$$

by the Wald's Identity. Hence, we get,

$$
\int_{0}^{\tau} \mathrm{E}|\tilde{k}(s)|^{2} d s=\frac{k_{0}^{2}}{\tilde{\sigma}^{2}}\left[\exp \left(\tilde{\sigma}^{2} \tau\right)-1\right] \text {. }
$$

Inserting this into (C.4) gives rise to,

$$
\mathrm{E}\left[\sup _{0 \leq t \leq \tau}\left|k\left(t \wedge \hat{\tau}_{\bar{W}}\right)-\tilde{k}\left(t \wedge \hat{\tau}_{\bar{W}}\right)\right|^{2}\right]
$$


$\leq 2 \sigma^{2} \int_{0}^{\tau} \mathrm{E}\left[\sup _{0 \leq t_{0} \leq s}\left|k\left(t_{0} \wedge \hat{\tau}_{\bar{W}}\right)-\tilde{k}\left(t_{0} \wedge \hat{\tau}_{\bar{W}}\right)\right|^{2}\right] d s+2 \xi^{2} \frac{k_{0}^{2}}{\tilde{\sigma}^{2}}\left[\exp \left(\tilde{\sigma}^{2} \tau\right)-1\right]$.

Hence, applying Gronwall's Inequality (see, Higham et al., 2003) yields,

$\mathrm{E}\left[\sup _{0 \leq t \leq T}\left|k\left(t \wedge \hat{\tau}_{\bar{W}}\right)-\tilde{k}\left(t \wedge \hat{\tau}_{\bar{W}}\right)\right|^{2}\right] \leq 2 \frac{k_{0}^{2}}{\tilde{\sigma}^{2}}\left[\exp \left(\tilde{\sigma}^{2} T\right)-1\right] \exp \left(2 \sigma^{2}\right) \xi^{2}$.

substituting this into (C.3) leads us to,

$\mathrm{E}\left[\sup _{0 \leq t \leq T}|k(t)-\tilde{k}(t)|^{2}\right] \leq 2 \frac{k_{0}^{2}}{\tilde{\sigma}^{2}}\left[\exp \left(\tilde{\sigma}^{2} T\right)-1\right] \exp \left(2 \sigma^{2}\right) \xi^{2}+\frac{2^{p+1} R W}{p}+\frac{2(p-2) W}{p R^{\frac{2}{p-2}} \bar{W}^{p}}$.

Accordingly, for $\forall \varepsilon>0$, we can choose $R$ and $\bar{W}$ such that,

$\frac{2^{p+1} R W}{p} \leq \frac{\varepsilon}{3}$ and $\frac{2(p-2) W}{p R^{\frac{2}{p-2}} \bar{W}^{p}} \leq \frac{\varepsilon}{3}$.

And for any given $T>0$, we put $\xi$ such that,

$2 \frac{k_{0}^{2}}{\tilde{\sigma}^{2}}\left[\exp \left(\tilde{\sigma}^{2} T\right)-1\right] \exp \left(2 \sigma^{2}\right) \xi^{2} \leq \frac{\varepsilon}{3}$.

Thus, for $\forall \varepsilon>0$, we obtain,

$\mathrm{E}\left[\sup _{0 \leq t \leq T}|k(t)-\tilde{k}(t)|^{2}\right] \leq \frac{\varepsilon}{3}+\frac{\varepsilon}{3}+\frac{\varepsilon}{3}=\varepsilon$.

So,

$\mathrm{E}\left[\sup _{0 \leq t \leq T}|k(t)-\tilde{k}(t)|^{2}\right] \rightarrow 0$ as $\varepsilon \rightarrow 0$.

i.e.,

$\lim _{T \rightarrow \infty} \mathrm{E}\left[\sup _{0 \leq t \leq T}|k(t)-\tilde{k}(t)|^{2}\right] \rightarrow 0$ as $\varepsilon \rightarrow 0$.

By the Levi Lemma, we obtain,

$\mathrm{E}\left[\lim _{T \rightarrow \infty} \sup _{0 \leq t \leq T}|k(t)-\tilde{k}(t)|^{2}\right] \rightarrow 0$ as $\varepsilon \rightarrow 0$.

which gives the desired result. And this proof is thus complete. 